\title{
Baseline Socio-Demographic and Mortality Profile of a Peri-urban Coastal Community in Karachi, Pakistan Before the Introduction of a Comprehensive Maternal, Newborn and Child Health Intervention Package
}

Muhammad llyas

Ministry of Public health

Kanwal Nayani

Mount Sinai Hospital

Ameer Muhammad

VITAL Pakistan Trust

Yasir Shafiq

VITAL Pakistan Trust

Benazir Baloch

Aga Khan University

Rehan Adamjee

VITAL Pakistan Trust

Fyezah Jehan

Aga Khan University

Imran Ahmed

Aga Khan University

Sajid Muhammad

Aga Khan University

Sajid Muhammad

Aga Khan University

Muhammad Imran Nisar ( $\nabla$ imran.nisar@aku.edu )

Aga Khan University

Research note

Keywords: Census, Socio-demographic characteristics, under-five mortality,

Posted Date: August 20th, 2021 
DOI: https://doi.org/10.21203/rs.3.rs-67851/v1

License: (c) (1) This work is licensed under a Creative Commons Attribution 4.0 International License. Read Full License 


\section{Abstract}

\section{Objective}

Pakistan has the highest neonatal mortality rate and one of the highest under- 5 mortality rates in the world, at 42 deaths and 74 deaths per thousand live births respectively. We undertook implementation of an evidence-based maternal, newborn and child health $(\mathrm{MNCH})$ intervention package to reduce under-five mortality in Rehri Goth, a peri-urban coastal community on the outskirts of Karachi, Pakistan. This paper aims to present the socio-demographic and under- 5 mortality profile of Rehri Goth prior to implementation of the intervention package. We conducted a detailed census of all households on sociodemographic variables.

\section{Results}

Over the course of the census period, 6,962 households were visited. The total population of Rehri Goth was found to be 42,980 . The male to female ratio was $52: 48$. Among adults aged 15 years and above, $67.1 \%$ had no formal education. The neonatal mortality and under-five mortality rates were 59 and 109 deaths per 1,000 live births respectively. Rehri Goth has a baseline child mortality rate that is higher than the national average in Pakistan. This provides an opportunity to deliver an evidence-based, targeted $\mathrm{MNCH}$ package to reduce child mortality.

\section{Introduction}

Globally, under-five mortality burden has fallen from 12.7 million in 1990 to almost 5.3 million in 2018. [1] The majority of these deaths have been averted by increasing coverage of simple, cost-effective interventions [2-5]. Perinatal complications and infections are amongst the top preventable causes of under-five mortality. $(3,5-8)$ About $45 \%$ of all child deaths are associated with malnourishment. $(7,9)$ Most of the deaths occur in Sub-Saharan Africa and South Asia, with about $50 \%$ deaths in China, Democratic Republic of the Congo, India, Nigeria and Pakistan. $(10,11)$

Pakistan's under-five mortality has fallen from 139 deaths per thousand live births in 1990 to 79 deaths per thousand live births in 2016, (11) however, progress has been insufficient in relation to other countries in the region and has consistently lagged Pakistan's gross domestic product per capita indicators. [12] This has resulted in Pakistan missing its Millennium Development Goals (MDGs) targets for under-five mortality. Pakistan now has the third highest burden of under-five deaths in the world, with 403,638 deaths occurring in 2018. (13) Poor health infrastructure, lack of skilled care during pregnancy and delivery, suboptimal referral system, low immunization coverage, under-nutrition, poor health-seeking 
behaviors, low literacy levels, and low status of women in society are leading causes contributing to the high under-five mortality rates in the country. $(14,15,16)$

In 2014, we began implementation of an evidence-based maternal, newborn and child health (MNCH) package to decrease under-five mortality in Rehri Goth, an impoverished peri-urban coastal community on the outskirts of Karachi. (17) This package included antenatal care and counselling for birth preparedness, promotion of skilled delivery, community health care for all newborns and infants, promotion of immunization, and family planning. (18) In this paper we aim to present the sociodemographic and child mortality profile of Rehri Goth prior to implementation of this evidence-based $\mathrm{MNCH}$ package. The child mortality estimates will serve as the baseline against which we will evaluate impact of our integrated package.

\section{Main Text}

\section{Study Site}

Rehri Goth is a fishing village spread over an area of 6.1 square kilometers and geographically divided into 45 different clusters. Each cluster comprises of an average of 250-300 structures, which are geospatially mapped. Department of Pediatrics and Child Health, the Aga Khan University has established a primary health center in the heart of community, which provides free of cost care to children under the age of five years. Figure $\mathrm{S} 1$ is map of the site.

\section{Study Design}

From March to April 2014, an independent consultant conducted a census of all households residing within the demarcated boundaries of Rehri Goth. A survey instrument was developed and translated into the local Urdu language for ease of administration. A 5-day training was conducted for 16 data collectors and 2 supervisors. Every household and person living in the community was enumerated. Information on age, gender and socio-demographic characteristics was collected. The supervisors checked all forms for completeness and consistency and sent forms with incomplete or inconsistent data back to the field for correction. Five percent of the households were revisited to validate the collected data. All the forms were double entered, and cross validation was done. All data were analyzed using Stata 12 and SPSS version 19.

\section{Results}

\section{Socio-demographic characteristics}

A total of 6,962 households were visited and 6,350 interviews were completed ( $91.2 \%$ response rate). The enumerated population was 42,980 with male to female ratio of 52:48. Figure 1 describes the population pyramid with age and gender distribution. Majority of the residents were Sindhi (51.8\%) followed by 
Pashto (16.9\%) and Urdu-speaking (9.1\%). Out of 24,149 adults aged $>15$ years, $68.3 \%$ were married. Only $8.1 \%$ reported that they treated water before drinking. Out of these, $56.9 \%$ boiled drinking water, while $33.6 \%$ strained it through a cloth. Only $41 \%$ of the participants reported that they wash hands with soap after defecation, while $35.6 \%$ washed hands before preparing meals and $34.6 \%$ washed hands before eating. Immunization rates were also low, with only $23 \%$ of the children aged 12-23 months completing all three doses of Pentavalent vaccine. Most of the births had taken place at home or at unskilled facility in the presence of a traditional birth attendant (57\%), with only $43 \%$ of births taking place at a skilled facility. Table 1 provides information on basic characteristics of the community. 
Table 1

Basic demographic and other characteristics of community

\begin{tabular}{|lc|}
\hline \multicolumn{2}{|l|}{ Ethnic distribution of the households $(\mathbf{n}=6350)$} \\
\hline Indicator & $\mathbf{N}(\%)$ \\
\hline Sindhi & $3291(51.8)$ \\
\hline Pashto & $1074(16.9)$ \\
\hline Urdu & $580(9.1)$ \\
\hline Bengali & $454(7.1)$ \\
\hline Punjabi & $413(6.5)$ \\
\hline Baluchi & $238(3.7)$ \\
\hline Hindko & $140(2.2)$ \\
\hline Saraiki & $101(1.6)$ \\
\hline Others & $59(0.9)$ \\
\hline Educational status of household members $(\mathbf{n}=21650)$ \\
\hline No Formal Education/ Illiterate & $16123(74.5)$ \\
\hline Pre -Primary (<5 Years) & $1146(5.3)$ \\
\hline Primary School (Up To 5 Years) & $1256(5.8)$ \\
\hline Middle & $969(4.5)$ \\
\hline Secondary & $1276(5.9)$ \\
\hline Higher Secondary & $599(2.8)$ \\
\hline Graduation and Above & $281(1.3)$ \\
\hline Hand washing practices after defecation $(\mathbf{n}=6350)$ \\
\hline With soap & $2602(41.0)$ \\
\hline Water only & $3474(54.7)$ \\
\hline Don't Wash & $274(4.3)$ \\
\hline
\end{tabular}

\section{Mortality Indicators}

There were 131 reported child deaths in 2013, based on prospective surveillance data from the project 'Aetiology of Neonatal Infections in South Asia (ANISA)', (19) and the census respectively. The Table 2 below shows data on live births, under-5 deaths, and mortality rates in Rehri Goth in 2013, with census 
data as a comparison. Furthermore, $55 \%$ of the deaths happened during $0-27$ days of the life, which highlights the need for targeted approaches to reduce neonatal and under-5 mortality. Table S1 is presenting percent breakdown of under-five deaths.

Table 2

Under-five death and mortality rate

\begin{tabular}{|lll|}
\hline & ANISA study 2012-2013 ${ }^{19}$ & Census 2014 \\
\hline Live births & 1206 & 1233 \\
\hline Indicators & $\mathbf{N}$ (rate) & $\mathbf{N}($ rate) \\
\hline Neonatal Deaths & $72(59.7)$ & $52(42.2)$ \\
\hline Infant Deaths & $110(91.2)$ & $91(73.8)$ \\
\hline Under-five Deaths & $131(108.6)$ & $114(92.5)$ \\
\hline
\end{tabular}

\section{Discussion}

The baseline under- 5 and neonatal mortality rates in Rehri Goth are 109 deaths and 59 deaths per thousand live births respectively, both of which are much higher than national average. Peri-urban slums such as Rehri Goth tend to have worse maternal and child health indicators than both rural and urban counterparts, given the poor reach of formal government healthcare and education services. $(17,20)$ There is an urgent need to develop service delivery models that are relevant for the peri-urban context. In light of this, the $\mathrm{MNCH}$ model that we propose to implement is comprehensive, coordinated, and integrated in nature, and targets the myriad of causes that contribute to the poor health status of periurban communities such as Rehri Goth, particularly focused on reducing the burden of neonatal deaths. It is our aim that demonstration of mortality reduction in such a community may provide a useful framework and operating model for effective service delivery in the peri-urban context.

The census reveals a population pyramid that is broader at the base and narrower at the top, which is common across developing country settings in the region and globally. Rates of immunization were significantly lower than national average, despite the presence of the Expanded Program of Immunization in the area. Many risk factors that lead to adverse health outcomes, such as low literacy percentage and poor hygiene behaviors, had high prevalence as reported in literature. (20) The strengths of this paper are that the survey had a high response rate $(>90 \%)$, was carried out by an independent survey organization, and employed local community health workers for data collection.

\section{Conclusion}

The high mortality rate in Rehri Goth will serve as the baseline against which the impact of the integrated $\mathrm{MNCH}$ program will be evaluated. 


\section{Limitations}

One of the limitations of census was recall bias. This might lead to under-estimation of the true mortality rate in the community. We were able to assess this by comparing census data with prospective surveillance data from the ANISA study, (19) which showed a mortality rate $15 \%$ higher than the census estimate. For purposes of evaluation, we will measure impact of our integrated $\mathrm{MNCH}$ package relative to the prospective surveillance mortality data.

\section{Abbreviations}

\begin{tabular}{|ll|}
\hline ANISA & Aetiology of Neonatal Infections in South Asia \\
\hline MDGs & Millennium Development Goals \\
\hline MNCH & Maternal, neonatal and child health \\
\hline
\end{tabular}

\section{Declarations}

\section{Ethics approval and consent to participate}

Approval was taken from community leaders and a locally representative non-governmental organization before starting the census and a verbal consent was taken from the head of each household before commencing the interview.

\section{Consent for publication}

Participants were informed during consent that de-identified aggregated results might be presented at different forums and published in scientific journals

\section{Availability of data and materials}

Data generated and analyzed during this census is available from the corresponding author on request.

\section{Competing interests}

None declared

\section{Funding}

The baseline census was funded by New World Foundation. The funders had no role in the design of the study, in collection, analysis, and interpretation of data, or in writing the manuscript.

\section{Acknowledgements}


Department of Pediatrics and Child Health at Aga Khan University, Data Management Unit Pediatric Research Office, field team, Community Health Workers, Rehri Health and Development Organization.

\section{Authors' contributions}

IN and MI led the design and implementation of the study. YS and BB helped in implementation. AKMZ provided technical oversight. IA and SM performed data analysis. MI and KN wrote the first draft. AM, RA, AKMZ and all other authors contributed to subsequent versions. All authors read and approved the final manuscript.

\section{References}

1. World Health Organization. Global Health Observatory (GHO) data 2018. [Accessed: August 18, 2019]; Available from: https://www.who.int/gho/child_health/mortality/mortality_under_five_text/en/

2. Kerber KJ, de Graft-Johnson JE, Bhutta ZA, Okong P, Starrs A, Lawn JE. Continuum of care for maternal, newborn, and child health: from slogan to service delivery. The Lancet. 2007 Oct 13; 370(9595):1358-69.

3. Bhutta ZA, Das JK, Rizvi A, Gaffey MF, Walker N, Horton S, Webb P, Lartey A, Black RE, Group TL, Maternal and Child Nutrition Study Group. Evidence-based interventions for improvement of maternal and child nutrition: what can be done and at what cost?. The lancet. 2013 Aug 3; 382(9890):452-77.

4. Lozano R, Wang H, Foreman KJ, Rajaratnam JK, Naghavi M, Marcus JR, Dwyer-Lindgren L, Lofgren KT, Phillips D, Atkinson C, Lopez AD. Progress towards Millennium Development Goals 4 and 5 on maternal and child mortality: an updated systematic analysis. The Lancet. 2011 Sep 24; 378(9797):1139-65.

5. Bryce J, Terreri N, Victora CG, Mason E, Daelmans B, Bhutta ZA, Bustreo F, Songane F, Salama P, Wardlaw T. Countdown to 2015: tracking intervention coverage for child survival. The Lancet. 2006 Sep 23;368(9541):1067-76.

6. Liu L, Johnson HL, Cousens S, Perin J, Scott S, Lawn JE, Rudan I, Campbell H, Cibulskis R, Li M, Mathers C. Global, regional, and national causes of child mortality: an updated systematic analysis for 2010 with time trends since 2000. The Lancet. 2012 Jun 9;379(9832):2151-61.

7. Bryce J, Boschi-Pinto C, Shibuya K, Black RE, WHO Child Health Epidemiology Reference Group. WHO estimates of the causes of death in children. The Lancet. 2005 Mar 26;365(9465):1147-52.

8. Wang $\mathrm{H}$, Liddell CA, Coates MM, Mooney MD, Levitz CE, Schumacher AE, Apfel H, lannarone M, Phillips B, Lofgren KT, Sandar L. Global, regional, and national levels of neonatal, infant, and under-5 mortality during 1990-2013: a systematic analysis for the Global Burden of Disease Study 2013. The Lancet. 2014 Sep 13;384(9947):957-79.

9. Black RE, Allen LH, Bhutta ZA, Caulfield LE, De Onis M, Ezzati M, Mathers C, Rivera J, Maternal and Child Undernutrition Study Group. Maternal and child undernutrition: global and regional exposures 
and health consequences. The lancet. 2008 Jan 19;371(9608):243-60..

10. Black RE, Morris SS, Bryce J. Where and why are 10 million children dying every year?. The lancet. 2003 Jun 28;361(9376):2226-34.

11. Oestergaard MZ, Inoue M, Yoshida S, Mahanani WR, Gore FM, Cousens S, Lawn JE, Mathers CD. Neonatal mortality levels for 193 countries in 2009 with trends since 1990: a systematic analysis of progress, projections, and priorities. PLoS medicine. 2011 Aug 30;8(8):e1001080.

12. You, D., Hug, L., Ejdemyr, S., Idele, P., Hogan, D., Mathers, C., Gerland, P., New, J.R. and Alkema, L., 2015. United Nations Inter-agency Group for Child Mortality Estimation (UN IGME). Global, regional, and national levels and trends in under-5 mortality between 1990 and 2015, with scenario-based projections to 2030: a systematic analysis by the UN Inter-agency Group for Child Mortality Estimation. Lancet, 386(10010), pp.2275-2286.

13. Nishtar S. Health indicators of Pakistan: gateway paper II. Heartfile; 2007.

14. UNICEF Data: Monitoring the situation of children and women 2018. (Source:https://data.unicef.org/country/pak/; accessed: March 14, 2019)

15. UNDP, Pakistan Millennium Development Goals. 2013, Ministry of Planning, Development and Reform, and United Nation Development Program: Islamabad.

16. Wagstaff A. Socioeconomic inequalities in child mortality: comparisons across nine developing countries. Bulletin of the World Health Organization. 2000;78:19-29.

17. Bhutta ZA, Hafeez A, Rizvi A, Ali N, Khan A, Ahmad F, Bhutta S, Hazir T, Zaidi A, Jafarey SN. Reproductive, maternal, newborn, and child health in Pakistan: challenges and opportunities. The Lancet. 2013 Jun 22;381(9884):2207-18.

18. Khan A, Kinney MV, Hazir T, Hafeez A, Wall SN, Ali N, Lawn JE, Badar A, Khan AA, Uzma Q, Bhutta ZA. Newborn survival in Pakistan: a decade of change and future implications. Health policy and planning. 2012 Jun 7;27(suppl_3):iii72-87.

19. Saha SK, Schrag SJ, El Arifeen S, Mullany LC, Islam MS, Shang N, Qazi SA, Zaidi AK, Bhutta ZA, Bose A, Panigrahi P. Causes and incidence of community-acquired serious infections among young children in south Asia (ANISA): an observational cohort study. The Lancet. $2018 \mathrm{Jul}$ 14;392(10142):145-59.

20. Save the Children. The Urban Disadvantage: State of the World's Mothers 2015.

\section{Figures}




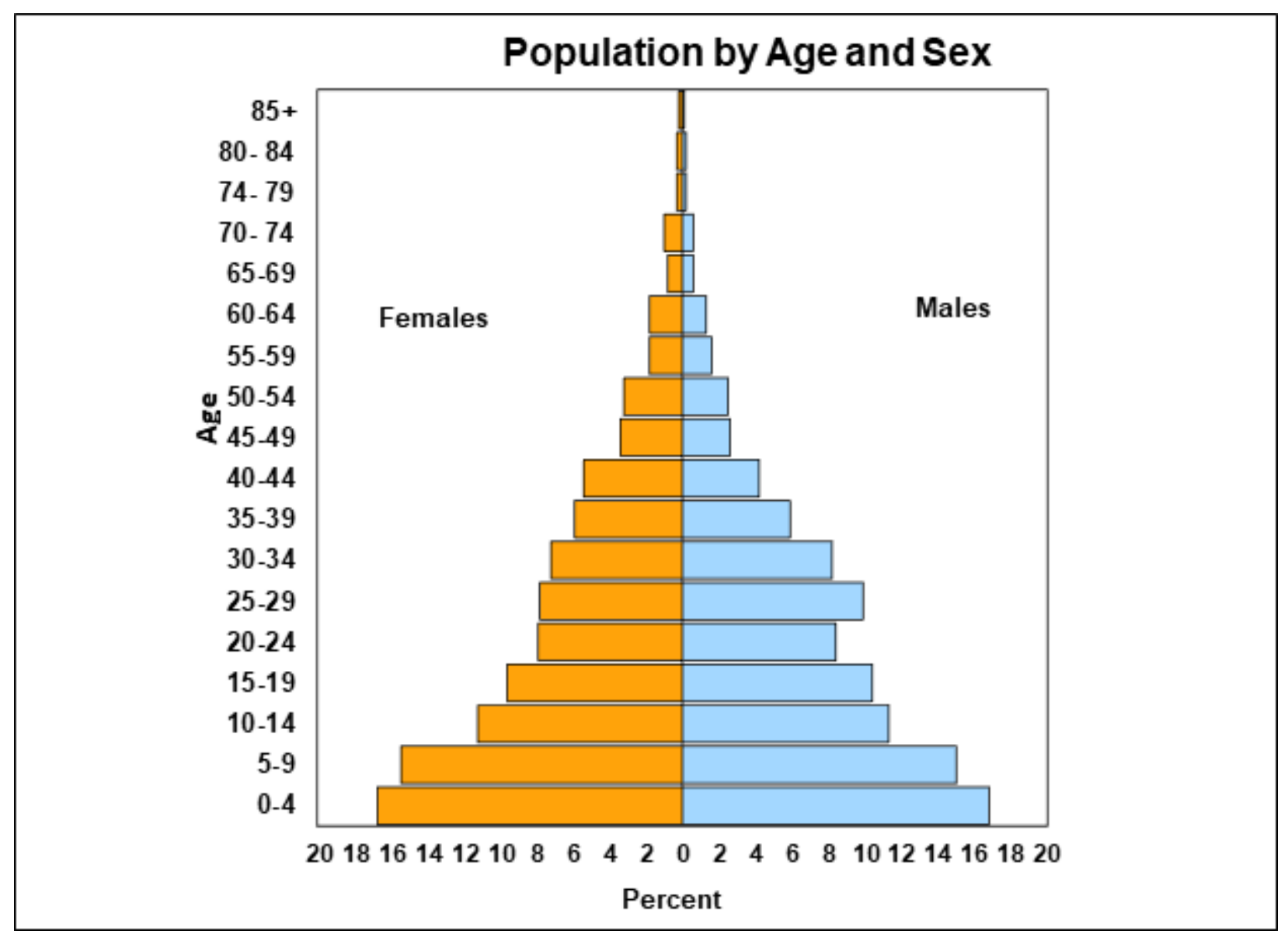

Figure 1

Population pyramid as per 2014 census

\section{Supplementary Files}

This is a list of supplementary files associated with this preprint. Click to download.

- Supplementaryfiguresandtables.docx 